\title{
Exercise training does not increase muscle FNDC5 protein or mRNA expression in pigs
}

\author{
John N. Fain ${ }^{1}$, Joseph M. Company², Frank W. Booth ${ }^{2,3,4}$, M. Harold Laughlin ${ }^{2,3,4}$, Jaume \\ Padilla $^{2}$, Nathan T. Jenkins ${ }^{2}$, Suleiman W. Bahouth ${ }^{5}$, and Harold S. Sacks ${ }^{6}$ \\ ${ }^{1}$ Department of Microbiology, Immunology and Biochemistry, College of Medicine, University of \\ Tennessee Health Science Center, Memphis Tennessee \\ ${ }^{2}$ Department of Biomedical Sciences, College of Veterinary Medicine, University of Missouri, \\ Columbia \\ ${ }^{3}$ Dalton Cardiovascular Research Center, University of Missouri, Columbia \\ ${ }^{4}$ Department of Medical Pharmacology and Physiology, College of Medicine, University of \\ Missouri, Columbia, Missouri \\ ${ }^{5}$ Department of Pharmacology, College of Medicine, University of Tennessee Health Science \\ Center, Memphis Tennessee \\ ${ }^{6}$ Endocrine and Diabetes Division, Veterans Greater Los Angeles Healthcare System and \\ Department of Medicine, UCLA, Los Angeles California
}

\section{Abstract}

Background-Exercise training elevates circulating irisin and induces the expression of the FNDC5 gene in skeletal muscles of mice. Our objective was to determine whether exercise training also increases FNDC5 protein or mRNA expression in the skeletal muscles of pigs as well as plasma irisin.

Methods-Castrated male pigs of the Rapacz familial hypercholesterolemic (FHM) strain and normal (Yucatan miniature) pigs were sacrificed after 16-20 weeks of exercise training. Samples of cardiac muscle, deltoid and triceps brachii muscle, subcutaneous and epicardial fat were obtained and FNDC5 mRNA, along with that of 6 other genes, was measured in all tissues of FHM pigs by reverse transcription polymerase chain reaction. FNDC protein in deltoid and triceps brachii was determined by Western blotting in both FHM and normal pigs. Citrate synthase activity was measured in the muscle samples of all pigs as an index of exercise training. Irisin was measured by an ELISA assay.

\footnotetext{
(C) 2013 Elsevier Inc. All rights reserved.

Author contributions as tissue sample collection and processing at the time of sacrifice.

Conflict of interest

The authors do not have any conflict of interest related to this manuscript.
}

The corresponding author is John N. Fain, Department of Microbiology, Immunology and Biochemistry, College of Medicine, University of Tennessee Health Science Center, Memphis Tennessee 38163 USA. jfain@uthsc.edu.

Publisher's Disclaimer: This is a PDF file of an unedited manuscript that has been accepted for publication. As a service to our customers we are providing this early version of the manuscript. The manuscript will undergo copyediting, typesetting, and review of the resulting proof before it is published in its final citable form. Please note that during the production process errors may be discovered which could affect the content, and all legal disclaimers that apply to the journal pertain.

JNF, MHL, SWB and HSS designed the study and oversaw its performance. JMC, JP, NYJ, FWB and JNF collected and analyzed data while JNF wrote the manuscript. JMC, FWB, MHL, JP and NTJ oversaw exercise training and supervision of the animals as well 
Results-There was no statistically significant effect of exercise training on FNDC5 gene expression in epicardial or subcutaneous fat, deltoid muscle, triceps brachii muscle or heart muscle. Exercise-training elevated circulating levels of irisin in the FHM pigs and citrate synthase activity in deltoid and triceps brachii muscle. A similar increase in citrate synthase activity was seen in muscle extracts of exercise-trained normal pigs but there was no alteration in circulating irisin.

Conclusion-Exercise training in pigs does not increase FNDC5 mRNA or protein in the deltoid or triceps brachii of FHM or normal pigs while increasing circulating irisin only in the FHM pigs. These data indicate that the response to exercise training in normal pigs is not comparable to that seen in mice.

\section{Keywords}

FNDC5; Exercise; Pigs; Muscle; Adipose tissue

\section{Introduction}

Recently attention has been paid to a protein called irisin that is derived from the extra cellular domain of fibronectin Type III domain-containing 5 protein [FNDC5] also known as FRCP2 [1-6]. FNDC5 has one trans-membrane spanning domain and the fibronectin III domain is one of three structural motifs found in fibronectin. Proteins containing this domain are thought to function as receptors or cell adhesion molecules and they can also influence neuronal development [1]. FNDC5/FRCP2 is strongly expressed in heart and skeletal muscle $[1,2]$. Irisin has been postulated to be a novel hormone released from skeletal muscle FNDC5 that mediates the beneficial effects of exercise on human adipose tissue in part by increasing the amount of UCP-1 in brown/beige adipocytes differentiated from precursors in white adipose tissue [3,4]. However, subsequent studies have shown that in humans, exercise programs induced only a small and transient increase in levels of circulating irisin [2] with no consistent increase in FNDC5 expression in muscle [5]. Polyzos et al [6] have reviewed the available information on FNDC5/irisin, which indicates that there are complex effects of this myokine in humans and much uncertainty as to whether it can account for the beneficial effects of exercise on human metabolic diseases. Swick et al [7] did find a high correlation between circulating levels of irisin and energy expenditure in postmenopausal overweight women, but only in those whose resting energy expenditure was not predicted by their fat free mass. As yet, there is no evidence that exercise in general elevates the circulating levels of irisin in humans [6].

Pigs are suitable animals for exercise studies because in a laboratory setting they are sedentary animals and both the intensity and duration of exercise-training on treadmills can be precisely regulated. Furthermore, unlike humans, the caloric intake can be kept constant in both sedentary and exercise-trained animals and their size is more comparable to humans than that of rodents. Furthermore due to their size, samples of epicardial fat and heart can be readily obtained. The present studies were designed to test the hypothesis that exercise training resulted in elevated circulating levels of irisin and enhanced FNDC5 mRNA expression and protein levels in skeletal muscles and heart of pigs. We also wanted to determine whether changes in FNDC5 expression in pig muscle are associated with changes in expression of mRNAs driving browning in mouse adipose tissue because this approach might provide insight into those genes in pigs where UCP-1 is non-functional $[8,9]$. Therefore, we measured mRNA for PRDM16 and PGC1a plus other thermogenesis-related genes [10-12] such as FGF 21, C/EBPa, HOXc8 and ZIC-1 in fat and muscle. 


\section{Methods}

\subsection{Approach}

This study was completed in two separate sets of pigs. The first were Rapacz FHM pigs (1011 months of age). These pigs have a defect in their low density lipoprotein receptor and exhibit hypercholesterolemia as well as extensive atherosclerosis of the coronary vessels [13]. We exercise trained 5 pigs from this group and compared results to 8 pigs not subjected to exercise training and have reported on epicardial fat gene expression in these pigs [13]. We considered the possibility that any effect of exercise could be the result of the hypercholesterolemia of the FHM pigs. To determine whether exercise training increased FNDC5 expression we also used archived skeletal muscle samples from normal (Yucatan) pigs from a previous study [14].

\subsection{Experimental animals. Protocol 1}

Before initiation of the study, approval was received from the Animal Care and Use Committee at the University of Missouri. Thirteen castrated adult male FHM pigs (10-11 months of age) were used in Protocol 1. The experimental design for the 16-20 week exercise training, collection of samples and RNA extraction were as previously reported [13]. Both the 8 unexercised and 5 exercised pigs were fed $800 \mathrm{~g} /$ day of the University of Wisconsin gestation diet (a cholesterol-free, 3\% fat, corn-soybean-based diet). All samples for assay were obtained at sacrifice, which occurred approximately 24 hours after the last exercise training session. Intramuscular ketamine/xylazine and intravenous pentothal was used to deeply anesthetize and euthanasia was achieved by heart extraction [13]. The hip SAT was removed from the hip region between the skin and the superficial muscles, coronary epicardial fat from the thick adipose tissue surrounding the proximal coronary vessels and myocardial epicardial fat from the thinner contiguous adipose tissue beginning 10 to $20 \mathrm{~mm}$ away from the coronary artery extending down on the ventricular myocardium.

\subsection{Experimental animals. Protocol 2}

Before initiation of the study, approval was received from the Animal Care and Use Committee at the University of Missouri. Sixteen adult male Yucatan miniature swine (normal pigs) purchased from a commercial breeder (Sinclair Research Farm, Columbia, MO) were used in Protocol 2. These pigs were 10-12 mo of age and weighed $25-40 \mathrm{~kg}$ at time of purchase. They were provided a standard diet (1,050 g/day of Purina Lab Mini-pig Chow) in which $8 \%$ of daily caloric intake was derived from fat. The normal pigs were divided into 8 exercise-trained and 8 non-exercise trained (sedentary) pigs [14].

The pigs were exercise trained by the same procedure in both protocols [13-14]. Pigs underwent exercise training treatment for 16-20 wk where sedentary pigs were restricted to their pens $(2 \times 4 \mathrm{~m})$ and exercised pigs performed a moderate-intensity $(70 \%$ of maximum heart rate) daily aerobic exercise-training program on treadmills one time per day, 5 days/ wk. Briefly, the aerobic exercise training protocol entailed a 5-min warm up at 2-2.5 miles per hour (mph), $15 \mathrm{~min}$ at $4 \mathrm{mph}$, and $20 \mathrm{~min}$ at $3 \mathrm{mph}$, and a $5 \mathrm{~min}$ cool-down at 2-2.5 $\mathrm{mph}$. The duration and intensity were increased each week so that by week 10 of training, the pigs performed a 5-min warm up at $2-2.5 \mathrm{mph}, 15 \mathrm{~min}$ at $6.5-7 \mathrm{mph}$, then $60 \mathrm{~min}$ at $4.5-5 \mathrm{mph}$, and finally a $5 \mathrm{~min}$ cool-down at $2-2.5 \mathrm{mph}$. Our laboratory [15-17] has established that this training program elicits the expected adaptations in exercise endurance and skeletal muscle oxidative capacity. For confirmation purposes, at the conclusion of the exercise training program, pigs performed a graded intensity treadmill exercise test to exhaustion. 
At time of death samples were taken from the 1) long head of triceps brachii, 2) deltoid muscles and 3) left ventricle of the heart. Muscle samples were frozen and stored at $-80^{\circ} \mathrm{C}$ until processed. Citrate synthase activity was measured from whole muscle homogenate of both groups of pigs using the spectrophotometric method of Srere [18].

\subsection{Protein analysis}

FNDC5 protein content was measured in skeletal muscle samples of the normal pigs using standard Western blot techniques as described previously (19). Briefly, frozen skeletal muscle samples were suspended in Laemmli buffer, boiled and sonicated to expose the intracellular contents. Total protein in each sample was measured with Bradford protein assay and $5 \mu \mathrm{g}$ loaded onto a polyacrylamide gel and separated by electrophoresis. After protein separation, contents of the gel were transferred to a polyvinylidene diflouride membrane by the application of $34 \mathrm{~V}$ for $1 \mathrm{~h}$, and this membrane was subsequently blocked with 5\% non-fat milk in TBS-Tween $(20 \mathrm{mM}$ Tris- $\mathrm{HCl}, 137 \mathrm{mM} \mathrm{NaCl}$, and $0.1 \%$ Tween 20) at room temperature for $1 \mathrm{~h}$. After $1 \mathrm{~h}$ incubation in non-fat milk, the primary antibody (Anti-FNDC5 antibody carboxyterminal end; ab93373 AbCam 1:1000) was applied overnight. The following morning the secondary antibody (rabbit-Sigma 1:2500) was applied for $1 \mathrm{~h}$. FNDC5 content was detected by enhanced chemiluminescence (Chemiluminescence kit - Pierce SuperSignal West Dura) and quantified by densitometry through the use of Kodak 4000R Imager and Molecular Imagery Software (Kodak Molecular Imaging Systems, New Haven, CT).

Plasma irisin concentrations were assayed in duplicate EDTA-treated plasma samples from FHM and normal pigs using an ELISA kit (Phoenix Pharmaceuticals) as recently described by our laboratory [20]. The intra-assay coefficient of variation was $2.5 \%$.

\subsection{Total RNA isolation}

Approximately 0.2 to $0.5 \mathrm{~g}$ of frozen fat or muscle from FHM pigs was homogenized with 5 $\mathrm{ml}$ of a monophasic solution of phenol and guanidine isothiocyanate (TRIzol reagent, Invitrogen, Carlsbad, CA). The frozen muscle samples were cut with scissors into small pieces prior to Polytron homogenization. The muscle samples were taken from the left ventricle of the heart, the long head of the triceps brachii and the deltoid muscle.

\subsection{RT-PCR}

The mRNA assay involved real-time quantitative PCR [21]. Transcriptor First Strand cDNA synthesis Kits from Roche Diagnostics were used on equal quantities of RNA to prepare the complementary DNA (cDNA). The Roche Lightcycler 480 Real-time RT-PCR system and Roche's Universal Probe Library of short hydrolysis Locked Nucleic Acid (LNA) dual hybridization probes combined with the primers recommended by their web-based assay design center [http://www.universalprobelibrary.com] were used for mRNA quantification. Integrated DNA Technologies (Coralville, IA) synthesized the primers. Seven mRNAs were targeted for their roles I) in muscle signaling to adipose tissue: FNDC5, PGC1a, FGF-21, C/EBPa, and 2) brown fat cell formation and thermogenesis: ZIC-1, PRDM16 and HOXc8.

In each assay, $55 \mathrm{ng}$ per tube of total RNA (determined by absorption at $260 \mathrm{~nm}$ in a nano spectrophotometer) was used. The data were obtained as $\mathrm{Cp}$ values obtained by the second derivative maximum procedure described in Roche Applied Science technical notes LC10/2000 and 13/2001 [http://www.roche-applied-science.com/sis/rtpcr/htc/index.jsp]. Relative quantification of the data was calculated using the absolute $\mathrm{Cp}$ values based on analyzing the same amount of total RNA in each assay as recommended by Bustin [22] and the relative amounts of mRNA were determined by the comparative $\mathrm{Cp}$ method, which removes the need for standard curves. This is the comparative $\mathrm{Ct}$ procedure expressed in the 
ABI PRISM 7700 Sequence Detection System user Bulletin 2 for quantitative RT-PCR. The calculation of ratios was done without an efficiency correction by assuming that the amount of target molecules doubles with every PCR cycle.

\subsection{Statistics}

A two-tailed Student $t$-test was performed to determine if differences between exercise and unexercised groups with respect to measurement of citrate synthase activity were significant at a $P$ value smaller than or equal to 0.05 since the Shapiro-Wilk test indicated that the values were normally distributed. A Mann-Whitney U nonparametric test was performed for all other comparisons and those differences with a $P$ value smaller than or equal to 0.05 were considered significant. Statistical analysis of mRNA values was based on the $\triangle \mathrm{Cp}$ values before $\log _{2}$ transformation.

\section{Results}

\subsection{Effects of exercise training on citrate synthase and FDNC5 protein content in skeletal muscle}

Sixteen to twenty weeks of exercise training climaxing by week 10 with 85 minutes a day on Monday-Friday, of castrated male FHM pigs resulted in enhanced levels of citrate synthase activity in the deltoid and triceps brachii muscles [Figure 1]. Since this effect of exercise may be the result of the hypercholesterolemia of the FHM pigs we also examined skeletal muscle samples from normal pigs but found the same increase in citrate synthase activity [Figure 1].

For studies on the effect of exercise training on FNDC5 protein expression we examined the deltoid and triceps brachii muscles [Figure 2]. Exercise training did not alter the amount of FNDC5 protein in deltoid or triceps brachii muscle of exercise trained FHM or normal pigs [Figure 2].

\subsection{Effect of exercise training on plasma irisin}

Plasma irisin was elevated by $42 \%$ in exercise-trained FHM as compared to sedentary pigs $(\mathrm{P}<0.05$, Fig 3). In contrast, plasma irisin concentrations were not different between exercise-trained and sedentary normal pigs (Figure 3).

\section{3. mRNA changes in skeletal and heart muscle of FHM pigs in response to exercise training}

Exercise training did not have a statistically significant effect on the mRNA level of FNDC5 in deltoid, triceps brachii or left ventricular heart muscle of FHM pigs [Table 1]. There was no effect of exercise training on the expression of any of the measured mRNAs in heart muscle. In contrast, exercise training reduced the expression of FGF21 and Hoxc8 in deltoid muscle [Table 1]. There were no effects of exercise training on the expression of PRDM16, $\mathrm{C} / \mathrm{EBPa}$, or ZIC-1 in any muscle. In the triceps brachii muscle of exercise-trained pigs there was a statistically significant increase in the expression of PGC1a. There were similar increases in deltoid and heart muscle of PGCla but they were not statistically significant.

\subsection{Effect of exercise training on mRNA levels in epicardial and hip subcutaneous fat of FHM pigs}

In epicardial fat of exercise-trained FHM pigs the only effect was a marked increase in PGC1a mRNA [Table 1]. There was no effect of exercise training on any of the mRNAs in hip subcutaneous fat of the FHM pigs [Table 1]. There were no effects of exercise training 
on the expression of FNDC5, FGF21, Hoxc8, PRDM16, C/EBPa, or ZIC-1 in subcutaneous or epicardial fat [Table 1].

The expression of most of the mRNAs in unexercised pigs was similar in subcutaneous and epicardial fat to what was seen in the muscles [Table 1]. Comparison of mRNA levels was based on comparing the $\mathrm{Cp}$ values in the different tissues for each mRNA. FNDC5 mRNA expression in heart was comparable to that in deltoid and triceps brachii muscle.

Furthermore there were only small differences, for the most part, between mRNA levels in skeletal as compared to heart muscle.

\subsection{Tissue mRNA correlations}

We examined the Pearson correlation coefficient between FNDC5 gene expression and that of the mRNAs for PRDM16, FGF21, C/EBPa and Hoxc8 in three fat depots [coronary epicardial, myocardial epicardial and hip SAT] and three muscles [deltoid, triceps brachii, left ventricular in both control and exercised FHM pigs [Figure 4]. In this figure we have plotted the group means for the control and exercise-trained pigs that are shown in Table 1 plus those for the myocardial epicardial fat. There was a very high correlation of 0.89 between FNDC5 and PRDM16 mRNA levels as well as a correlation of 0.71 for Hoxc8 and of 0.70 for C/EBPa. The correlation coefficient was 0.49 between FNDC5 and FGF21 gene expression but it was not statistically significant.

The Pearson correlation in the deltoid and triceps brachii muscle of each of the 13 FHM pigs ( 8 controls and 5 exercise-trained pigs) between FNDC5 expression in deltoid muscle or Triceps brachii muscle and that of the mRNAs shown in Table 1 was also investigated. There was no correlation of FNDC5 gene expression in the skeletal muscles with any of these mRNAs. In contrast, FNDC5 expression in heart muscle significantly correlated with that of ZIC-1 [ $n=13, r=0.63$ and $p=0.02]$. In fat there was no correlation of FNDC5 gene expression with any mRNA except PRDM16 $[\mathrm{n}=13, \mathrm{r}=0.56$ and $\mathrm{P}=0.045]$ in subcutaneous adipose tissue and of PPAR $[\mathrm{n}=13, \mathrm{r}=0.72$ and $\mathrm{p}=0.006]$ in epicardial adipose tissue.

\section{Discussion}

Contrary to our hypothesis, neither FNDC5 protein nor mRNA was up regulated in skeletal muscle of exercise trained pigs. The change in muscle FNDC5 gene expression required to cause detectable elevations in circulating irisin may be so small it cannot be detected. Another possibility that needs to be acknowledged is that exercise training might have affected a post-translational step in irisin expression such as cleavage of FNDC5 to produce irisin. We did see a significant (42\%) increase in circulating irisin in the exercise trained FHM pigs but this was not observed in normal pigs suggesting that exercise-training does not consistently increase circulating irisin, or that the genetic dyslipidemia and atherosclerotic complications in FHM influence plasma irisin concentrations. There were similar effects of exercise training on citrate synthase activity of deltoid and triceps brachii indicating that the rise of circulating irisin in FHM pigs is associated with exercise training in FHM pigs. The elevation in circulating irisin without any change in FNDC5, its precursor protein, in deltoid or triceps brachii indicates that the rise of irisin in FHM pigs comes from another source or that the change in muscle FNDC5 required to cause detectable elevations in circulating irisin is so small that they do not result in any alteration in its gene or protein expression.

None of the data cited above rule out an important role for FNDC5 in muscle energy metabolism. This is because, in pigs, uncoupling protein-1 [UCP-1] is non-functional $[8,9]$ which may account for the difference between our results and those observed in mice and 
humans. For this reason we did not examine UCP-1 gene expression. The lack of functional UCP-1 in pigs does not seem to have deleterious effects. Newborn pigs are not especially cold sensitive and pigs engage in nesting behavior to keep piglets relatively warm [23]. It is difficult to conceive any situation except severe cold exposure where increased thermogenesis in skeletal muscle would be of adaptive value. The converse of this is that organisms with higher energy efficiency due to a lack of UCP-1 might be able to deposit more fat and muscle per kilo of food. The role of UCP-1 in mediating brown fat thermogenesis due to catecholamines or cold exposure in rodents is well established. However, the role of UCP-1 in thermogenesis in adult humans is still being investigated [24].

PGC1a is known to play a major role in adaptive thermogenesis of brown fat. It may also play an even more important role in the adaptations to exercise where it is a key mediator of many of the beneficial effects of exercise training on skeletal muscle physiology [25]. We found increases in PGCla mRNA in all tissues after exercise training but they were statistically significant only in epicardial fat and triceps brachii muscle. The lack of correlation between PGC1a and FNDC5 mRNA levels in pig muscles could be linked to the lack of functional UCP-1 [8,9]. A strong correlation [coefficient $r$ of 0.94 ] was reported by Huh et al [2] between FNDC5 and PGC1a mRNA in human skeletal muscle. In pigs PGC1a mRNA is elevated at least 30-fold in heart or skeletal muscle over fat but what it means is unknown except that this was not accompanied by any significant increase in expression of FNDC5 protein. It is also unclear why comparable amounts of FNDC5 mRNA are found in pig muscle and fat tissue.

In heart muscle, exercise training did not alter FNDC5 mRNA or, for that matter, any other mRNA that we examined. Previously we found that exercise training increased the heart weight in four out of 5 of the same exercised-trained FHM pigs [13]. Because our individual mRNA data are expressed per unit of total RNA, the possibility exists that exercise training caused an increase in the total amount of RNA in the heart and proportionate increases in the mRNAs examined.

The PRDM16 gene was examined since it has been reported to control the shifting of myoblasts into brown fat cells [10]. Clearly the expression of PRDM16 mRNA is not linked to that to PGC1a whose expression in the three muscles was far higher than was seen in the fat depots. There is a key role for PRDM16 in the myoblast to brown fat switch [10,11] and this may well translate into an enhanced thermogenesis in muscle of pigs but if so is unrelated to UCP-1. The present results clearly differentiate pig from human muscle since in human skeletal muscle a correlation coefficient of 0.94 between FNDC5 mRNA and that of PGC1a was seen [2] but in pig muscle FNDC5 expression correlates with that of PRDM16.

FGF 21 was also examined because it has been reported to alter expression of thermogenic genes in fat tissue [12]. ZIC-1 is a member of a conserved family of zinc finger proteins with a role in axial skeletal patterning in vertebrate embryos and regulates Myf5 activation in somites [26]. Myf5 is a gene whose expression is involved in brown fat differentiation and PRDM16 is said to control the brown fat to skeletal muscle switch [11]. The role of Hoxc8 in muscle function is unclear. This protein is a homeodomain transcription factor that interacts with Smad proteins to activate osteopontin gene expression in response to bone morphogenetic proteins $[27,28]$.

Two markers of adipose tissue browning in humans, which is defined as the conversion of white fat into brown fat or an intermediate form of fat known as beige fat, are HOXc8 and ZIC1 $[4,29,30]$. The mRNA for HOXc-8 is down in brown/beige fat while that of ZIC-1 is up $[4,29,30]$. By this definition the EAT in pigs was more brown-like since the expression of 
HOXc8 was down but there was no change in ZIC-1 as compared to SAT. However, in exercise-trained FHM pigs there was no significant change in the expression of HOXc8 in either fat depot but there was a significant decrease in HOXc8 expression in deltoid but not T. brachii or heart muscle. There was a high correlation between HOXc8 expression and that of FNDC5 in all fat and muscle depots tested in both control and exercise-trained FHM pigs. The significance of this correlation is unclear as well as the fact that this was seen to the same extent in both fat and muscle.

A strength of this report is the use of pigs as an experimental model for exercise-training since the exercise and food consumption can be readily regulated which is difficult to do with human studies. It was possible to obtain samples of heart muscle, which cannot be done in exercise-trained humans. Among the shortcomings of the use of pigs is the lack of functional UCP-1 protein, which means that irisin effects on energy metabolism of pigs must involve different signaling mechanisms. Another shortcoming is that we did not examine the effect of a short burst of exercise or other adaptive changes in the pigs but rather samples were obtained $24 \mathrm{~h}$ after the last of the 16-20 week training sessions. The present data suggest that, in pigs, irisin does not link exercise to thermogenesis in fat hence alternative hypotheses should be considered not involving irisin.

In conclusion, neither pig skeletal muscle FNDC5 protein or mRNA expression was altered by chronic exercise training in normal or FHM pigs. However, circulating levels of irisin are elevated by exercise training but only in FHM pigs.

\section{Acknowledgments}

The authors acknowledge the technical assistance of Paramjeet Cheema, Pam Thorne and Dave Harah as well as statistical analysis by Jim Keras.

Funding This study was supported by NIH-NHLBI Grants HL52490, and HL36088 to MHL and by a contract from the Cardiometabolic Disease Research Foundation of Los Angeles, CA to JNF. NTJ was supported by NIHT32 AR048523.

\section{Abbreviations}

$\begin{array}{ll}\text { FNDC5 } & \text { fibronectin Type III domain-containing } 5 \text { protein } \\ \text { PRDM16 } & \text { PR-domain-missing 16, PGC1a, PPARY- coactivator-1 } \\ \text { UCP-1 } & \text { uncoupling protein-1 } \\ \text { FHM pigs } & \text { Rapacz Familial Hypercholesterolemic (FHM) pigs } \\ \text { hip SAT } & \text { hip subcutaneous fat } \\ \text { EAT } & \text { epicardial fat } \\ \text { mEAT } & \text { myocardial epicardial fat } \\ \text { cEAT } & \text { coronary epicardial fat } \\ \text { RTPCR } & \text { reverse transcriptase polymerase chain reaction } \\ \text { FGF21 } & \text { fibroblast growth factor 21 } \\ \text { C/EBPa } & \text { CCAAT/enhancer binding protein } \\ \text { Cp } & \text { crossing point values; }\end{array}$




\section{References}

1. Teufel A, Malik N, Mukopadhyay M, et al. Frcp1 and Frcp2, two novel fibronectin type III repeat containing genes. Gene. 2002; 297(1-2):79-83. [PubMed: 12384288]

2. Huh JY, Panagiotou G, Mougios V, et al. FNDC5 and irisin in humans: I. Predictors of circulating concentrations in serum and plasma and II. mRNA expression and circulating concentrations in response to weight loss and exercise. Metabolism. 2012; 61(12):1725-1738. [PubMed: 23018146]

3. Bostrom P, Wu J, Jedrychowski MP, et al. A PGC1-a-dependent myokine that drive brown-fat-like development of white fat and thermogenesis. Nature. 2012; 481(7382):463-468. [PubMed: 22237023]

4. Spiegelman BM. Banting lecture 2012: regulation of adipogenesis: toward new therapeutics for metabolic disease. Diabetes. 2013; 62(6):1774-1782. [PubMed: 23704518]

5. Timmons JA, Baar K, Davidsen PK, et al. Is irisin a human exercise gene? Nature. 2012; 488(7413):E9-E10. [PubMed: 22932392]

6. Polyzos SA, Kountouras J, Shields K, Mantzoros CS. Irisin: A renaissance in metabolism? Metabolism. 2013 http://dx.doi.org/10.1016/j.metabol.2013.04.008.

7. Swick AG, Orena S, O'Connor A. Irisin levels correlate with energy expenditure in a subgroup of humans with energy expenditure greater than predicted by fat free mass. Metabolism. $2013 \mathrm{http} / / /$ dx.doi.org/10.1016/j.metab.2013.02.012.

8. Trayhurn P, Temple NJ, Van Aerde J. Evidence from immunoblotting studies on uncoupling protein that brown adipose tissue is not present in the domestic pig. Can J Physiol Pharmacol. 1989; 67(12): 1480-1485. [PubMed: 2627687]

9. Berg F, Gustafson U, Andersson L. The uncoupling protein 1 gene (UCP1) is disrupted in the pig lineage: a genetic explanation for poor thermoregulation in piglets. PLoS Genet. 2006; 2(8):11781181.

10. Seale P, Bjork B, Yang W, et al. PRDM16 controls a brown fat/skeletal muscle switch. Nature. 2008; 454(7207):961-967. [PubMed: 18719582]

11. Kajimura S, Seale P, Kubota K, et al. Initiation of myoblast to brown fat switch by a PRDM16-C/ EBP-a transcriptional complex. Nature. 2009; 460(7259):1154-1158. [PubMed: 19641492]

12. Fisher FM, Kleiner S, Douris N, et al. FGF21 regulates PGC-1 and browning of white adipose tissues in adaptive thermogenesis. Genes Dev. 2012; 26(3):271-281. [PubMed: 22302939]

13. Company JM, Booth FW, Laughlin MH, et al. Epicardial fat gene expression after aerobic exercise training in pigs with coronary atherosclerosis: relationship to visceral and subcutaneous fat. J Appl Physiol. 2010; 109(6):1904-1912. [PubMed: 20947714]

14. Padilla J, Simmons GH, Davis JW, et al. Impact of exercise training on endothelial transcriptional profiles in healthy swine a genome-wide microarray analysis. Am J Physiol Heart Circ Physiol. 2011; 301(2):H555-H564. [PubMed: 21622830]

15. Muller JM, Myers PR, Laughlin MH. Vasodilator responses of coronary arterioles from pigs with early-stage coronary disease induced by high-fat, high-cholesterol diet: effect of exercise. J Appl Physiol. 2004; 97(3):1159-1168. [PubMed: 15208294]

16. Padilla J, Newcomer SC, Simmons GH, et al. Long-term exercise training does not alter brachial and femoral artery vasomotor function and endothelial phenotype in healthy pigs. Am J Physiol Heart Circ Physiol. 2010; 299(2):H379-H385. [PubMed: 20511414]

17. Thompson MA, Henderson KK, Woodman CR, et al. Exercise preserves endothelium-dependent relaxation in coronary arteries of hypercholesterolemic male pigs. J Appl Physiol. 2004; 96(3): 1114-1126. [PubMed: 14617527]

18. Srere PA. Citrate synthase. Methods Enzymol. 1969; 13:3-5.

19. Laughlin MH, Pollock JS, Amann JF, Hollis ML, et al. Training Induces Non-uniform Increases in eNOS content along the Coronary Arterial Tree. J Appl Physiol. 2001; 90(2):501-510. [PubMed: 11160048]

20. Roberts MD, Bayless D, Childs TC, Company JM, Jenkins NT, Padilla J, Martin J, Dalbo V, Booth FW, Rector RS, Laughlin MH. Irisin precursor FNDC5 mRNA is elevated in skeletal muscle during obesity in OLETF rats. Metabolism. $2013 \mathrm{http}: / / d x . d o i . o r g / 10.1016 / j . m e t a b$. 2013.02.0002. 
21. Sacks HS, Fain JN, Holman B, et al. Uncoupling protein-1 and related messenger ribonucleic acids in human epicardial and other adipose tissues: epicardial fat functioning as brown fat. J Clin Endocrinol Metab. 2009; 94(9):3611-3615. [PubMed: 19567523]

22. Bustin SA. Absolute quantification of mRNA using real-time reverse transcription polymerase chain reaction assays. J Mol Endocrinol. 2000; 25(2):169-193. [PubMed: 11013345]

23. Algers B, Uvnas-Moberg K. Maternal behavior in pigs. Horm Behav. 2007; 52(1):78-85. [PubMed: 17482189]

24. van Marken Lichtenbelt WD, Schrauwen P. Implications of nonshivering thermogenesis for energy balance regulation in humans. Am J Physiol Regul Integr Comp Physiol. 2011; 301(2):R285R296. [PubMed: 21490370]

25. Handschin C, Spiegelman BM. The role of exercise and PGC1a in inflammation and chronic disease. Nature. 2008; 454(7203):463-469. [PubMed: 18650917]

26. Pan H, Gustafsson MK, Aruga J, et al. A role for Zic1 and Zic2 in Myf5 regulation and somite myogenesis. Dev Biol. 2011; 351(1):120-127. [PubMed: 21211521]

27. Bai S, Shi X, Yang X, et al. Smad6 as a transcriptional corepressor. J Biol Chem. 2000; 275(12): 8267-8270. [PubMed: 10722652]

28. Yang X, Ji X, Shi X, et al. Smad1 domains interacting with Hoxc-8 induce osteoblast differentiation. J Biol Chem. 2000; 275(2):1065-1072. [PubMed: 10625647]

29. Cheung L, Gertow J, Werngren O, et al. Human mediastinal adipose tissue displays certain characteristics of brown fat. Nutr Diabetes. 2013; 13(3):e66. [PubMed: 23670224]

30. Jespersen NZ, Larsen TJ, Peijs L, et al. A classical brown adipose tissue mRNA signature partly overlaps with brite in the supraclavicular region of adult humans. Cell Metab. 2013; 17(5):798805. [PubMed: 23663743] 


\section{FHM pigs}

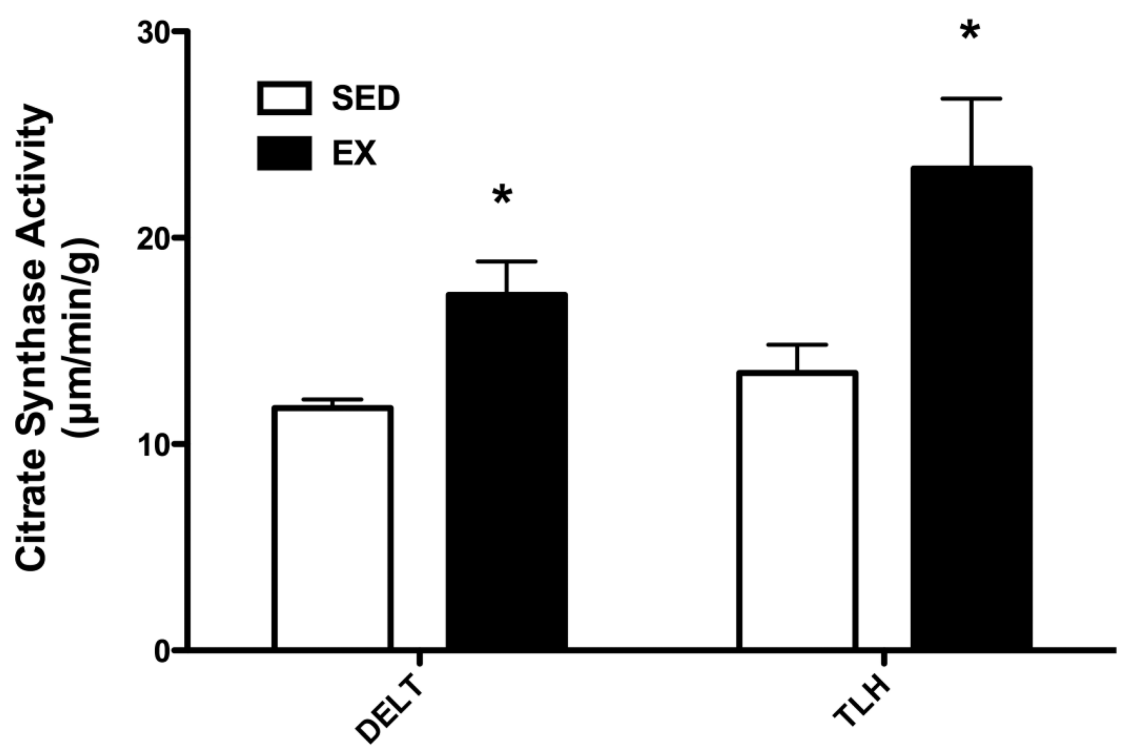

Normal pigs

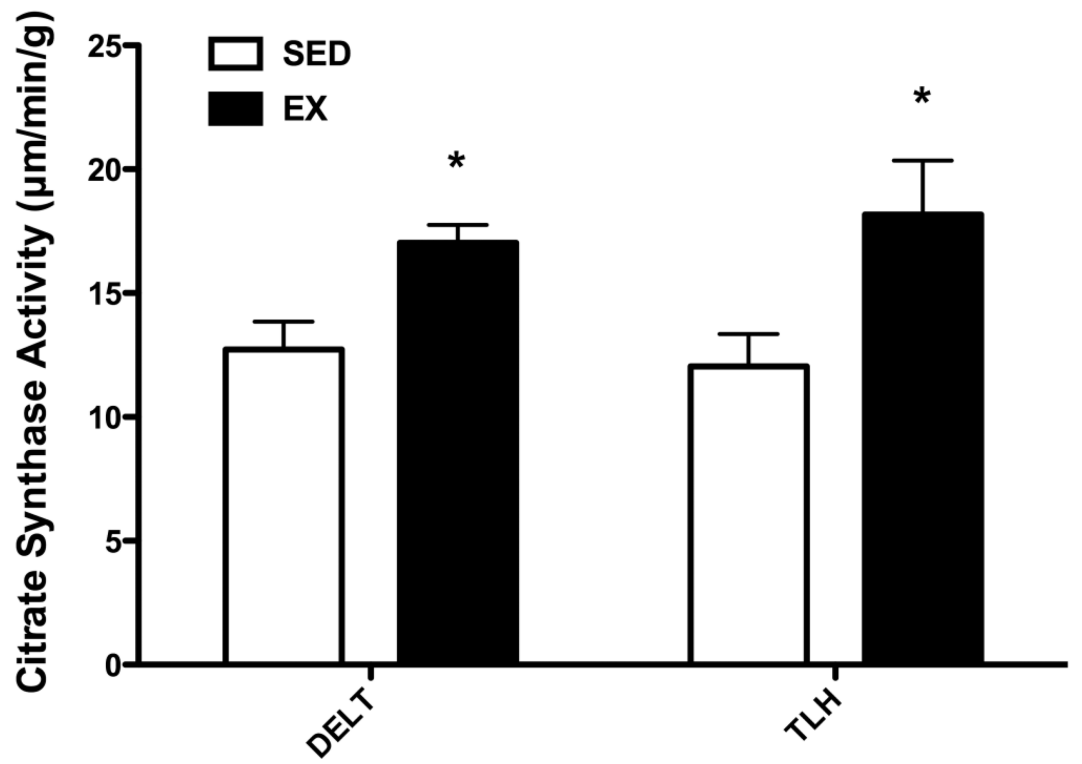

Figure 1.

Citrate synthase activity for the exercise trained and sedentary FHM pigs. The data for pigs that did not train on the treadmill (SED) and for the exercised trained (EX) pigs for deltoid muscle (DELT) and long head of the triceps brachii (TLH) muscles of FHM pigs are shown in the top part while those for normal pigs are shown in the bottom part of the figure. Means $\pm \mathrm{SEM}$ are presented (FHM SED $=8, \mathrm{EX}=5$; Normal Pigs $\mathrm{n}=8$ in both SED and EX groups) for samples that were obtained at sacrifice. Note that exercise training induced similar increases in citrate synthase activity in both FHM and normal pigs. * indicates that the EX value is significantly greater ( $P$ less than 0.05 ) than the SED value for that muscle. 


\section{FHM pigs}
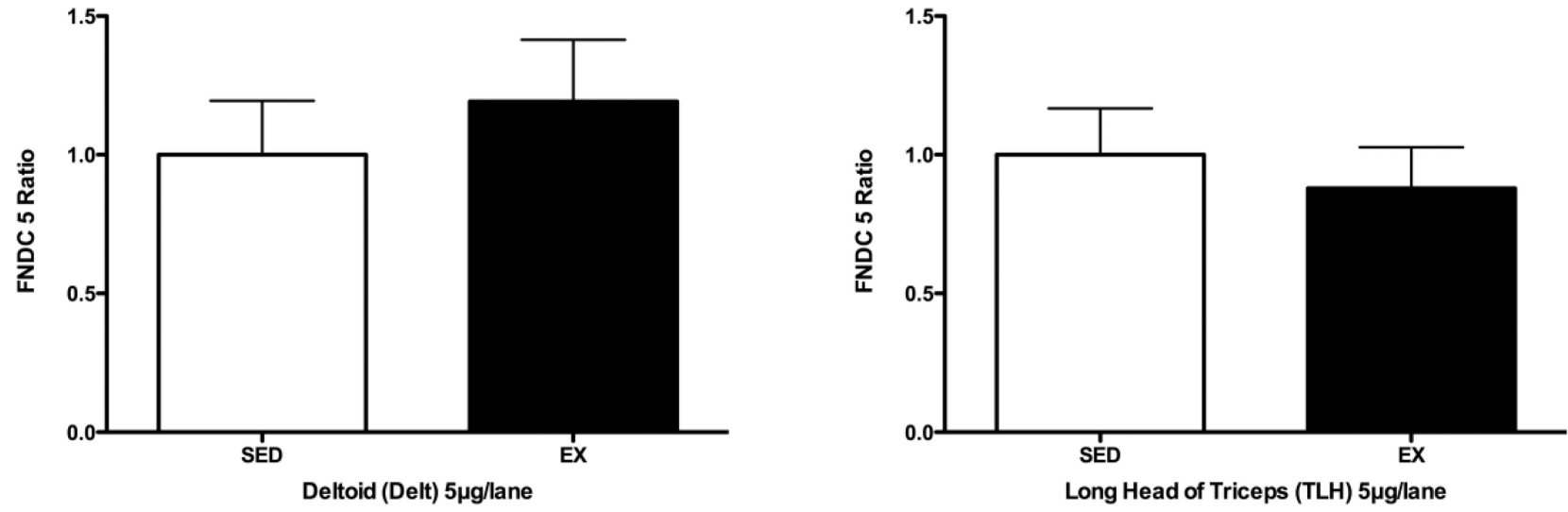

$\begin{array}{lllllllll}S & E & E & S & S & E & E & S\end{array}$

Blot 3

Blot 4
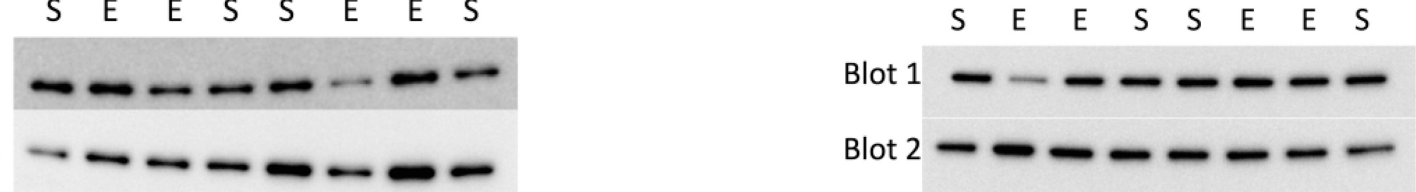

\section{Normal pigs}
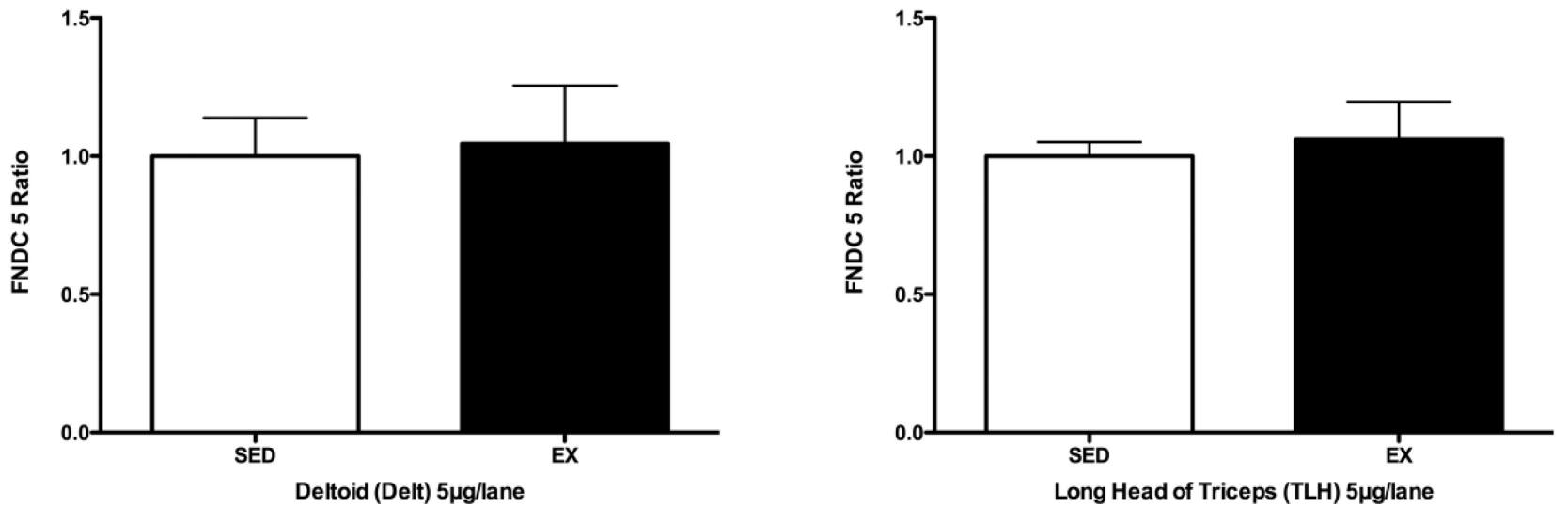

Figure 2.

Immunoblot results for FNDC5 immuonreactivity in deltoid and long head of the triceps brachii muscles of normal and FHM pigs. The data are shown as the ratio of the means of immunoreactivity in exercise trained (EX) relative to that of FHM pigs that did not train on the treadmill (SED). The data in the top part of the figure are for 5 exercise-trained and 8 sedentary FHM pigs while the bottom shows data for 8 exercise-trained and 8 sedentary normal pigs for samples that were obtained at sacrifice. The SED values on each blot were averaged and then the data on that blot were expressed relative to the SED mean value. The blots for each muscle sample used to calculate the means of the normal pigs are also shown. 


\section{FHM pigs}

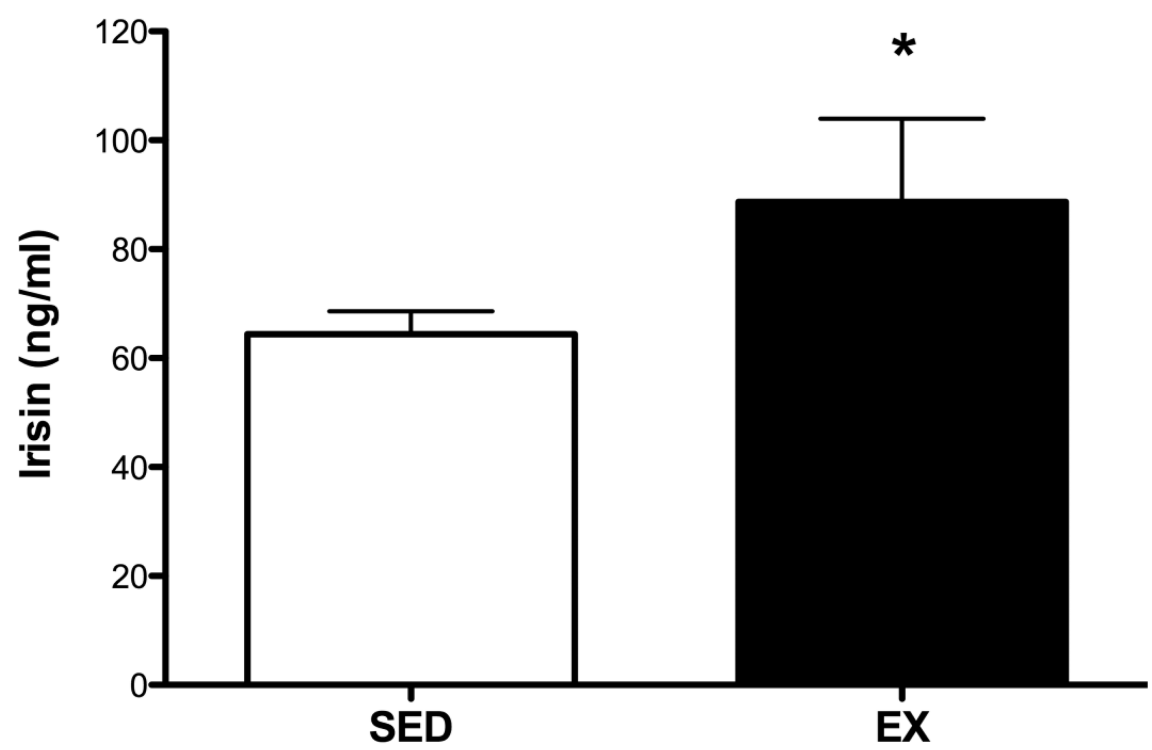

\section{Normal pigs}

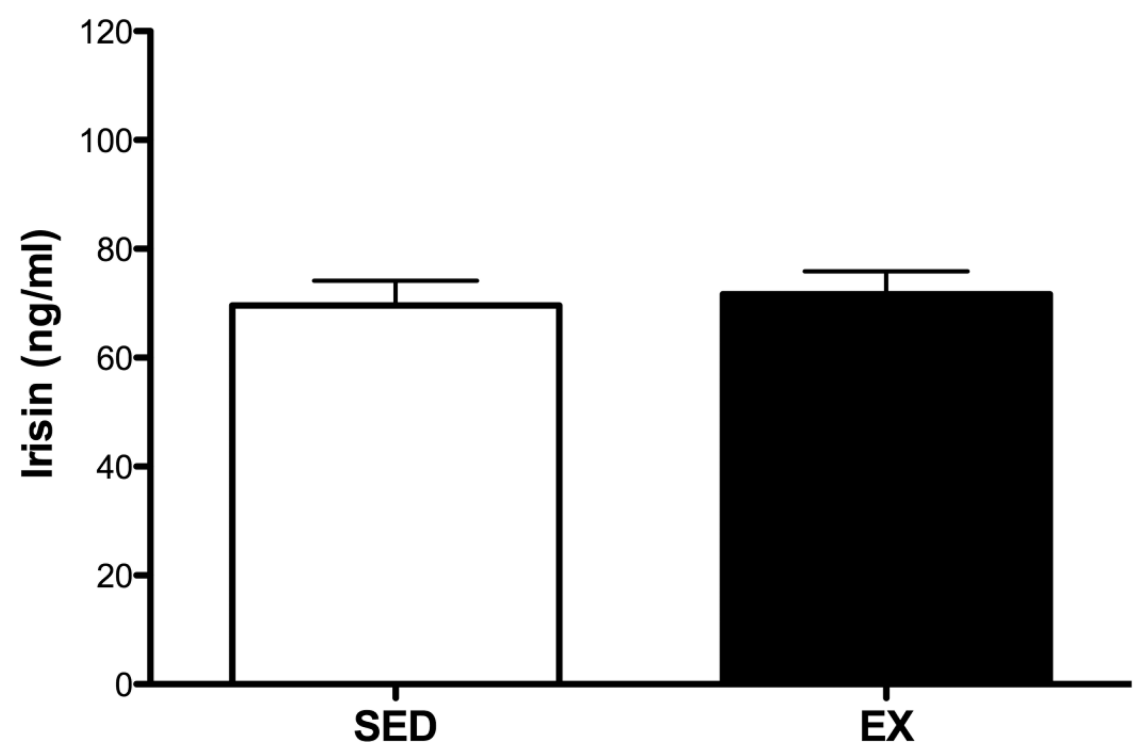

Figure 3.

Plasma irisin in exercise-trained and sedentary pigs. The data in the top part of the figure are for 5 exercise-trained and 8 sedentary FHM pigs while the bottom shows data for 8 exercisetrained and 8 sedentary normal pigs for samples that were obtained at sacrifice. $*$ indicates that the exercise-trained value is significantly greater ( $\mathrm{P}$ less than 0.05$)$ than the control/ sedentary value. 
A $\mathrm{n}=12$ and $\mathrm{r}=0.71$ with $\mathrm{P}=0.01$

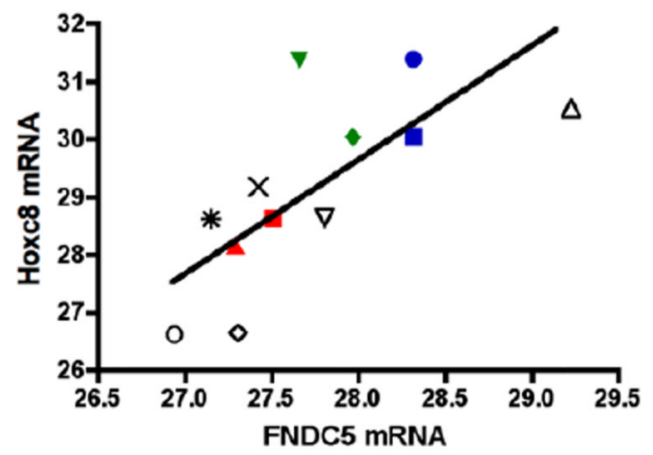

- Hip SAT/ex

- Hip SAT/sed

$\nabla$ cEAT/ex

- cEAT/sed

- mEAT/ex

- mEAT/sed

$\triangle$ Deltoid/ex

$\nabla$ Deltoid/sed

$\diamond$ T. brachii/ex

- T. brachii/sed

$x$ heart LV/ex

* heart LV/sed

C $n=12$ and $r=0.70$ with $P=0.01$

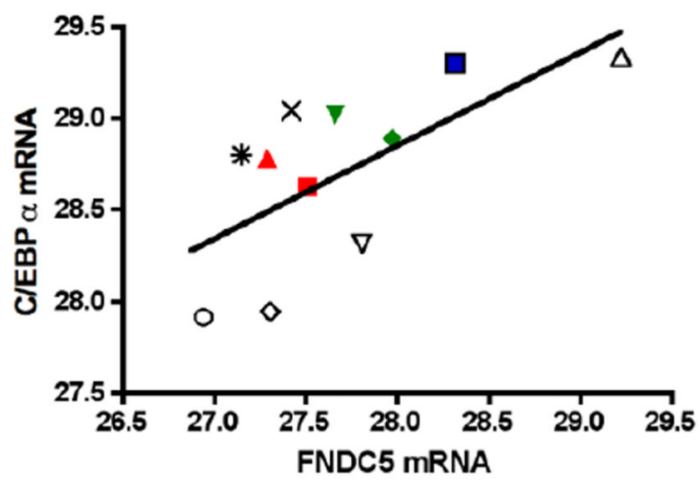

- Hip SAT/ex

- Hip SAT/sed

$\checkmark$ cEAT/ex

- cEAT/sed

- mEAT/ex

- mEAT/sed

$\Delta$ Deltoid/ex

$\nabla$ Deltoid/sed

$\diamond$ T. brachii/ex

- T. brachii/sed

$X$ heart LV/ex

* heart LV/sed

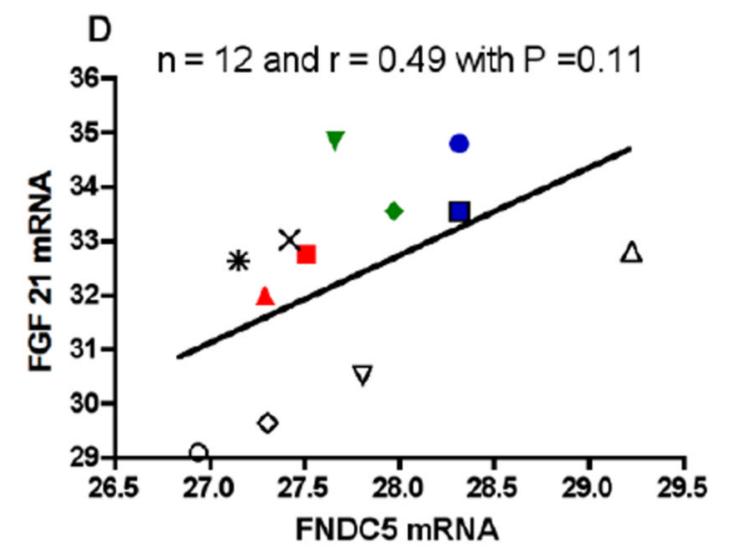

Figure 4.

Correlation between mRNA expression of FNDC5 and that of PRDM16, FGF21, CEBP and Hoxc8 in fat and heart muscle of Rapacz FHM pigs. The Pearson correlation coefficient [r] was calculated between the group means for the six tissues in coronary epicardial fat [cEAT], myocardial epicardial fat [mEAT], hip subcutaneous fat [Hip SAT], deltoid muscle [Deltoid], triceps brachii [T. brachii] and left ventricular heart muscle [heart LV] in both unexercised [sed] and exercised [ex] pigs. The group means for all except myocardial epicardial fat are also shown in Table 1. The data in part A are for HOXc8, part B for PRDM16, part C for C/EBPa and part D for FGF21. 


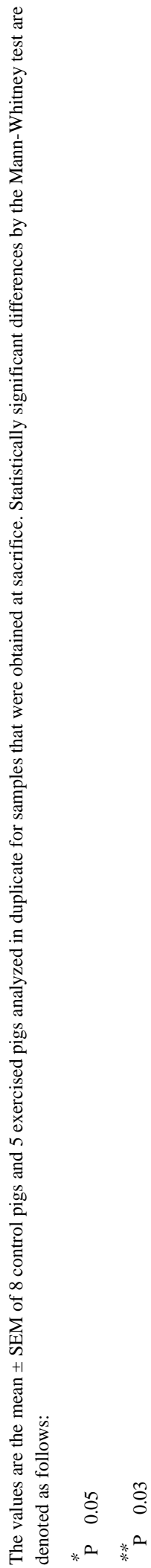

